\title{
GEOMETRICALLY NONLINEAR FREE VIBRATIONS OF FUNCTIONALLY GRADED BEAM WITH DISCONTINUITIES RESTING ON NONLINEAR ELASTIC FOUNDATIONS
}

\author{
CHAJDI Mohcine ${ }^{1 \mathrm{a}}$, MERRIMI EI bekkaye ${ }^{1 \mathrm{~b}}$, and EL BIKRI Khalid ${ }^{1 \mathrm{c}}$ \\ ${ }^{1}$ Mohammed V University in Rabat, ENSET - Rabat, LaMIPI, B.P.6207, Rabat Instituts, Rabat, Mo- \\ rocco \\ achajdimohcine@gmail.com, ${ }^{b}$ merbe2004@yahoo.fr, ck.elbikri@um5s.net.ma
}

Keywords: Nonlinear vibration, Functionally graded beam, Crack, Porosities, Elastic foundation.

\begin{abstract}
This paper studies the geometrically nonlinear free vibration characteristics of functionally graded beam clamped at both ends with an edge crack and including uniform porosities resting on an elastic foundation. The theoretical formulations is based on Euler-Bernoulli beam theory and von Karman geometric nonlinearity assumptions. A semi-analytical model based on Hamilton's principle and spectral analysis combined with a homogenisation method is used to reduce the problem under consideration to that of an equivalent isotropic homogeneous cracked beam resting on an elastic foundation. A modified rule of mixture, taking the effect of porosities into account, is adopted in evaluating the effective material properties and assumed to be varying continuously in the thickness direction according to a simple power law distribution in terms of the volume fractions of the constituents. The problem is solved by a numerical iterative method which investigates the effects of crack depth, material property gradient, foundations parameters, on dynamic response of cracked functionally graded beam.
\end{abstract}

(C) 2017 The Authors. Published by Eccomas Proceedia.

Peer-review under responsibility of the organizing committee of COMPDYN 2017.

doi: $10.7712 / 120117.5485 .18181$ 


\section{INTRODUCTION}

Functionally graded structures have found and won its applicability in various fields such as automobiles, space vehicles, defence industries and aerospace engineering because of their remarkable advantages. One of the most applicable structures as basic mechanical elements in structural analysis for the modelling of many engineering structures are beams. FGM beams are often subjected to vibrations operating under a large number of random cyclic stresses and destructive effects in the form of initial defects in the material or due to stress concentration or high level working loads. As a result, it is natural to expect that cracks to occur in the structural elements. It is known that a crack in a structural element causes local variations in its rigidity, increases its vulnerability to static and dynamic loads. Therefore, understanding the mechanical behaviour and safe performance of cracked FGM structures is very important in design. In fact, extensive research has been conducted on the dynamic behaviour of FGM beams using different methods. Ke et al [1] studied the nonlinear vibration of cracked FGM beams using differential quadrature method. Yang and Chen [2] discussed the influence of open edge cracks on the vibration of Euler-Bernoulli FGM beams with different boundary conditions using analytical method. T. Yan et al [3] studied the nonlinear dynamic response of an edge-cracked functionally graded Timoshenko beam under parametric excitation using Rung-Kutta and multiple scale methods. In addition, the effect of porosities on the mechanical behaviour of FGM structures has been considered in recent years. In the manufacture of FGM, micro-voids or porosities may occur in the materials during the sintering process due to the large difference in the temperatures of solidification between the constituents of the material. Therefore, it is important to consider the effect of porosity when designing FGM structures. The problem of vibration of the FGM porous structures has been studied by some authors such as Chen et al [4] studied free and forced vibrations of shear deformable functionally graded porous beams, they employed Ritz method and Newmark- $\beta$ method. S. Yousef et al [5] investigated free vibration of functionally Euler-Bernoulli and Timoshenko graded porous beams using transfer matrix method. In some engineering applications, for instance railway lines, geotechnical areas, construction structures and offshore structures, beams are often attached to elastic foundations that exert reaction forces or moments depending on Displacements or rotations. In the literature, there are two parameters for modelling the foundation, for a single parameter, there is the model called Winkler, the second is the Pasternak model, as the second parameter is related to the stiffness of the shear of the foundation. However, this motivated many researchers to analyse the mechanical behaviour of FG beams resting on elastic foundation. The effect of the elastic foundation on the natural frequencies and dynamic behaviour of FGM beams has been investigated by the following researchers, T. Yan et al [6] studied the dynamic response of FGM beam with an open edge crack resting on an elastic foundation subjected to a transverse load moving at a constant speed they are employed Galerkin's procedure and Newmark- $\beta$ method. M. Arefi [7] evaluated the nonlinear responses of FGM beam resting on a nonlinear foundation using ADM method. S. Akbas et al [8] studied the free vibration analysis of an edge cracked functionally graded cantilever beam resting on Winkler-Pasternak foundation using finite element method, and later they studied a free vibration and static bending analysis of FGM beam resting on Winkler foundations using the Navier-type solution and exact formulas [9]. A. Fallah et al [10] compared the variational method with other analytical methods for the nonlinear natural frequency and post-buckling load-deflection relationship of the FGM beam on nonlinear elastic foundation. $\mathrm{H}$. Yaghoobu et al [11] studied a large amplitude vibration and post-buckling behaviour of geometrically imperfect functionally graded beams resting on nonlinear foundation with simply supported and clamped-clamped boundary conditions. The objective of the present work is to determine the geometrically nonlinear free vibration of cracked functionally graded beam with 
porosities resting on nonlinear elastic foundation, using a semi-analytical method. The beam thickness was supposed constant and the FGM properties are assumed to vary through the beam thickness. In this study, a homogenisation procedure is developed and used to reduce the problem under consideration to that of an equivalent isotropic homogeneous cracked beam resting on nonlinear elastic foundation. Upon assuming harmonic motion, the discretized expressions for the total strain and kinetic energies of the beam are then derived, and by applying Hamilton's principle, the problem is reduced to a system of coupled nonlinear algebraic equations. Direct iterative method described in [12] is employed for solving the eigenvalue equation for governing the frequency nonlinear vibration, in order to show the effect of the crack depth, the influences of the porosity volume fraction and foundation stiffness parameters, on dynamic response of cracked functionally graded beams.

\section{THEORY AND FORMULATIONS}

\subsection{Mechanical properties of the FGM beam with porosities}

Consider a FGM beam made from a mixture of ceramic-metal materials including porosity phases, containing an edge crack of depth $a$ located at a distance $x_{0}$ from the left end, resting on Winkler-Pasternak foundation having the geometrical characteristics shown in Fig. 1.

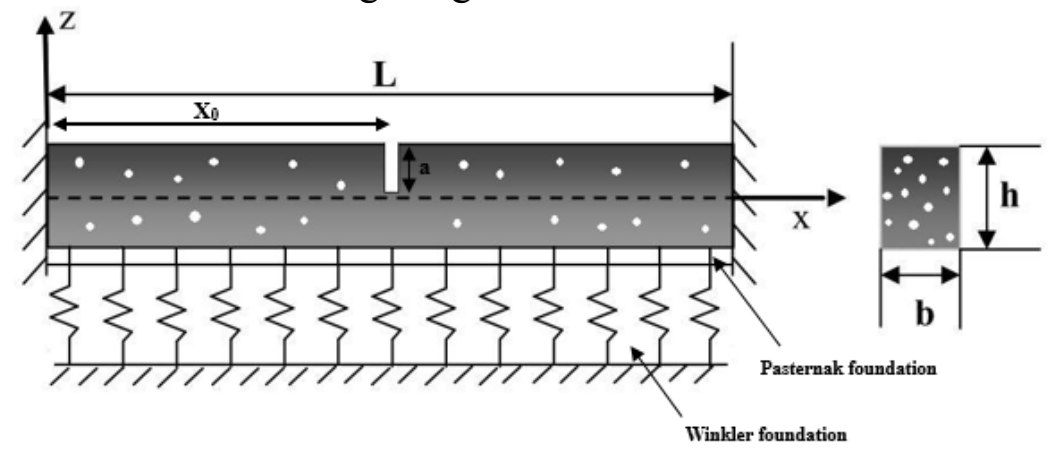

Figure 1: Physical model of the cracked FGM beam with porosities resting on Winkler-Pasternak foundation.

The effective material properties of the cracked FGM beam such as Young's modulus $E$, and mass density $\rho$, with porosity volume fraction $\zeta(\zeta<<1)$, are assumed that vary continuously through the thickness of the beam, according to power law form.

$$
P(z)=P_{m}\left(\vartheta_{m}-\frac{\zeta}{2}\right)+P_{c}\left(\vartheta_{c}-\frac{\zeta}{2}\right)
$$

Where $P_{m}$ and $P_{c}$, are the material properties of the metal and the ceramic constituents respectively.

In which $\vartheta_{\mathrm{c}}$ and $\vartheta_{\mathrm{m}}$ are the ceramic and metal volume fractions, respectively and are related as follows:

$$
\vartheta_{m}+\vartheta_{c}=1
$$

The power law of volume fraction of the ceramic $\vartheta_{\mathrm{c}}$ is defined by

$$
\vartheta_{c}(z)=\left(\frac{z}{h}+\frac{1}{2}\right)^{k} \quad k \geq 0
$$


The gradient index $k$ is the non-negative variable parameter (power-law exponent) which determines the material distribution across the beam thickness and $z$ is the distance from the mid-plane of the FGM beam.

Hence, all properties of the imperfect FGM beam can be written as follows:

$$
P(z)=\left(P_{c}-P_{m}\right)\left(\frac{z}{h}+\frac{1}{2}\right)^{k}+P_{m}-\left(P_{c}-P_{m}\right) \frac{\zeta}{2}
$$

\section{NONLINEAR FREE VIBRATION ANALYSIS OF CLAMPED-CLAMPED CRACKED BEAMS RESTNING ON ELASTIC FOUNDATION}

\subsection{Total strain and kinetic energy expressions of cracked FGM beam}

The total strain energy $V_{T}$ of the cracked FGM beam is given as the sum of the strain energy due to the bending $V_{b}$, the stored axial strain energy to large amplitudes $V_{a}$, the stored strain energy at the crack location $V_{c}$ and The strain energy of the elastic foundation $V_{f}$.

$$
V_{T}=V_{b}+V_{a}+V_{c}+V_{f}
$$

Where:

$$
\begin{gathered}
V_{a}=\frac{(E S)_{\text {eff }}}{8 l}\left(\int_{0}^{l}\left(\frac{\partial W}{\partial x}\right)^{2} d x\right)^{2} \\
V_{b}=\frac{(E I)_{e f f}}{2}\left(\int_{0}^{l}\left(\frac{\partial^{2} W}{\partial x^{2}}\right)^{2} d x\right) \\
V_{c}=\frac{\left((E I)_{e f f}\right)^{2}}{2 K_{f}}\left(\frac{\partial^{2} W}{\partial x^{2}}\right)_{x=x_{0}} \\
V_{f}=\frac{1}{2} \int_{0}^{l}\left(k_{L} W^{2}+\frac{1}{2} k_{N L} W^{4}+K_{s}\left(\frac{\partial W}{\partial t}\right)^{2}\right) d x
\end{gathered}
$$

The expression of the kinetic energy $T$ neglecting the axial and rotational inertia can be written as:

$$
T=\frac{(\rho S)_{e f f}}{2} \int_{0}^{l}\left(\frac{\partial W}{\partial t}\right)^{2} d x
$$

Where:

$$
\begin{gathered}
(E S)_{\text {eff }}=b A_{11} \\
(E I)_{\text {eff }}=b\left(D_{11}-\frac{B_{11}}{A_{11}}\right) \\
(\rho S)_{\text {eff }}=\int_{-\frac{h}{2}}^{\frac{h}{2}} \rho(z) d z
\end{gathered}
$$


Where $A_{11}, B_{11}$ and $D_{11}$ are the extension-extension, bending-extension, bending-bending coupling rigidity coefficients respectively.

\subsection{Governing equation}

It is well known that the dynamic behaviour for a conservative system may be obtained by application of Hamilton's principle which can write as:

$$
\delta \int_{t 1}^{t 2}(T-V) d t=0
$$

As the movement is harmonic, the transverse displacement is written as:

$$
W(x, t)=a_{i}(t) W_{i}(x)=a_{i} W(x) \sin \omega t
$$

For a general parametric study, the following dimensionless formulation is used:

$$
x^{*}=\frac{x}{L}, W_{i}^{*}=\frac{W_{i}(x)}{r}
$$

Where $r=\sqrt{\frac{I}{S}}$

Applying Hamilton's principle and expanding the displacement $W$ in the form of a finite series, the following set of nonlinear amplitude equations is obtained:

$$
a_{i} K_{i r}^{*}+\frac{3}{2} a_{i} a_{j} a_{k} B_{i j k r}^{*}-\omega^{* 2} a_{i} M_{i r}^{*}=0
$$

Where $K_{i r}^{*}, B_{i j k r}^{*}$ and $M_{i r}^{*}$ stand for the dimensionless classical rigidity tensor, the nonlinear rigidity tensor and the mass tensor, respectively, which are defined as:

$$
\begin{gathered}
K_{i j}^{*}=\int_{0}^{l} \frac{\partial^{2} W_{i}^{*}(x)}{\partial x^{* 2}} \frac{\partial^{2} W_{j}^{*}(x)}{\partial x^{* 2}} d x^{*}+\left.\left.\beta \frac{\partial^{2} W_{i}^{*}(x)}{\partial x^{* 2}}\right|_{x_{0}^{*}} \frac{\partial^{2} W_{j}^{*}(x)}{\partial x^{* 2}}\right|_{x_{0}^{*}}+k_{L}^{*} \int_{0}^{l} W_{i}^{*}(x) W_{j}^{*}(x) d x^{*}+k_{S}^{*} \int_{0}^{l} \frac{\partial W_{i}^{*}(x)}{\partial x^{*}} \frac{\partial W_{j}^{*}(x)}{\partial x^{*}}(x) d x^{*} \\
B_{i j k l}^{*}=\alpha\left(\int_{0}^{l} \frac{\partial W_{i}^{*}(x)}{\partial x^{*}} \frac{\partial W_{j}^{*}(x)}{\partial x^{*}} d x^{*}\right)\left(\int_{0}^{l} \frac{\partial W_{k}^{*}(x)}{\partial x^{*}} \frac{\partial W_{l}^{*}(x)}{\partial x^{*}} d x^{*}\right)+k_{N L}^{*} \int_{0}^{l} W_{i}^{*}(x) W_{j}^{*}(x) W_{k}^{*}(x) W_{l}^{*}(x) d x^{*} \\
M_{i j}^{*}=\int_{0}^{l} W_{i}^{*}(x) W_{j}^{*}(x) d x^{*}
\end{gathered}
$$

Where:

$$
\begin{gathered}
\alpha=\frac{r^{2}}{4} \frac{(E S)_{\text {eff }}}{E I_{\text {eff }}} \quad \beta=\frac{(E I)_{\text {eff }}}{K_{f} L} \\
k_{L}^{*}=\frac{k_{L} l^{4}}{(E I)_{\text {eff }}} \quad k_{N L}^{*}=\frac{k_{N L} l^{4} r^{2}}{2(E I)_{\text {eff }}} \quad k_{s}^{*}=\frac{k_{s} l^{2}}{(E I)_{\text {eff }}}
\end{gathered}
$$

Where $k_{L}$ and $k_{N L}$ are linear and nonlinear elastic foundation coefficients, respectively, and $k_{S}$ is the coefficient of shear stiffness of the elastic foundation. 


\section{NUMERICAL RESULTS AND DISCUSSION}

To test the validity and accuracy of the method used in this study with the published data, the frequency ratios, calculated in the present work at various vibration amplitudes and various value of volume fraction $k$, is compared with the results obtained in [10]. The material properties applied to nonlinear verification analysis of FGM beams $\left(E_{c}=330.2 \mathrm{GPa}, \rho_{c}=2370 \mathrm{Kg} / \mathrm{m}^{3}\right.$, $\left.E_{m}=207.8 \mathrm{GPa}, \rho_{m}=8166 \mathrm{Kg} / \mathrm{m}^{3}\right)$.

\begin{tabular}{|ccccccc|}
\hline \multirow{2}{*}{$\boldsymbol{W}_{\text {max }}^{*}$} & \multicolumn{2}{c}{$\mathrm{k}=1$} & & & $\mathrm{k}=2$ & \\
\cline { 2 - 7 } & Present & {$[10]$} & Error $(\%)$ & Present & {$[10]$} & Error (\%) \\
\hline 0 & 1.000 & 1.000 & 0.0 & 1.000 & 1.000 & 0.0 \\
1 & 1.055 & 1.055 & 0.0 & 1.054 & 1.054 & 0.0 \\
2 & 1.208 & 1.205 & 0.3 & 1.202 & 1.200 & 0.2 \\
3 & 1.426 & 1.418 & 0.8 & 1.415 & 1.409 & 0.6 \\
4 & 1.685 & 1.669 & 1.6 & 1.668 & 1.656 & 1.2 \\
\hline
\end{tabular}

Table 1: Comparison of frequency ratios of a C-C FGM beam at various amplitudes for $k_{l}^{*}=k_{n l}^{*}=k_{s}^{*}=0$.

In Table 1, the frequency ratio for C-C FGM beam is listed. A reasonably good agreement with previous results for nonlinear analysis can be observed.

Also, to demonstrate the accuracy of our solution method, the frequency ratio of the C-C FGM beam compared with those reported in [11]. It is observed that the present results agree very well with those given by [11].

\begin{tabular}{|cccc|}
\hline & $\boldsymbol{W}_{\boldsymbol{m} \boldsymbol{m a x}}^{*}=0.5$ & & \\
\cline { 2 - 4 } $\mathrm{k}$ & Present & {$[11]$} & Error $(\%)$ \\
\hline 0 & 1.03919354 & 1.04011944 & 0.092 \\
\cline { 2 - 4 } 1 & 1.03931242 & 1.04035038 & 0.10 \\
\hline 2 & 1.03905031 & 1.03990573 & 0.08 \\
\cline { 2 - 4 } 3 & 1.03888136 & 1.03961710 & 0.07 \\
\hline 4 & 1.03921999 & 1.03946159 & 0.02 \\
\hline & 1.03881148 & 1.03938366 & 0.05 \\
\hline
\end{tabular}

Table 2: Comparison of the effect of the gradient index $k$ on the frequency ratio of the C-C FGM beam for

$$
\left(k_{l}^{*}=50 \quad k_{n l}^{*}=50 \quad k_{s}^{*}=5\right) \text {. }
$$

On the other hand for the following results, others material properties are considered, the top surface of the FGM is ceramic rich $\left(E_{c}=350 \mathrm{GPa}, \rho_{c}=3800 \mathrm{Kg} / \mathrm{m}^{3}\right)$, whereas the bottom surface of the FGM is metal rich $\left(E_{m}=200 \mathrm{GPa}, \rho_{m}=2780 \mathrm{Kg} / \mathrm{m}^{3}\right)$. The beam is supposed to be cracked at its mid-span and only the fundamental nonlinear mode shape is considered. 


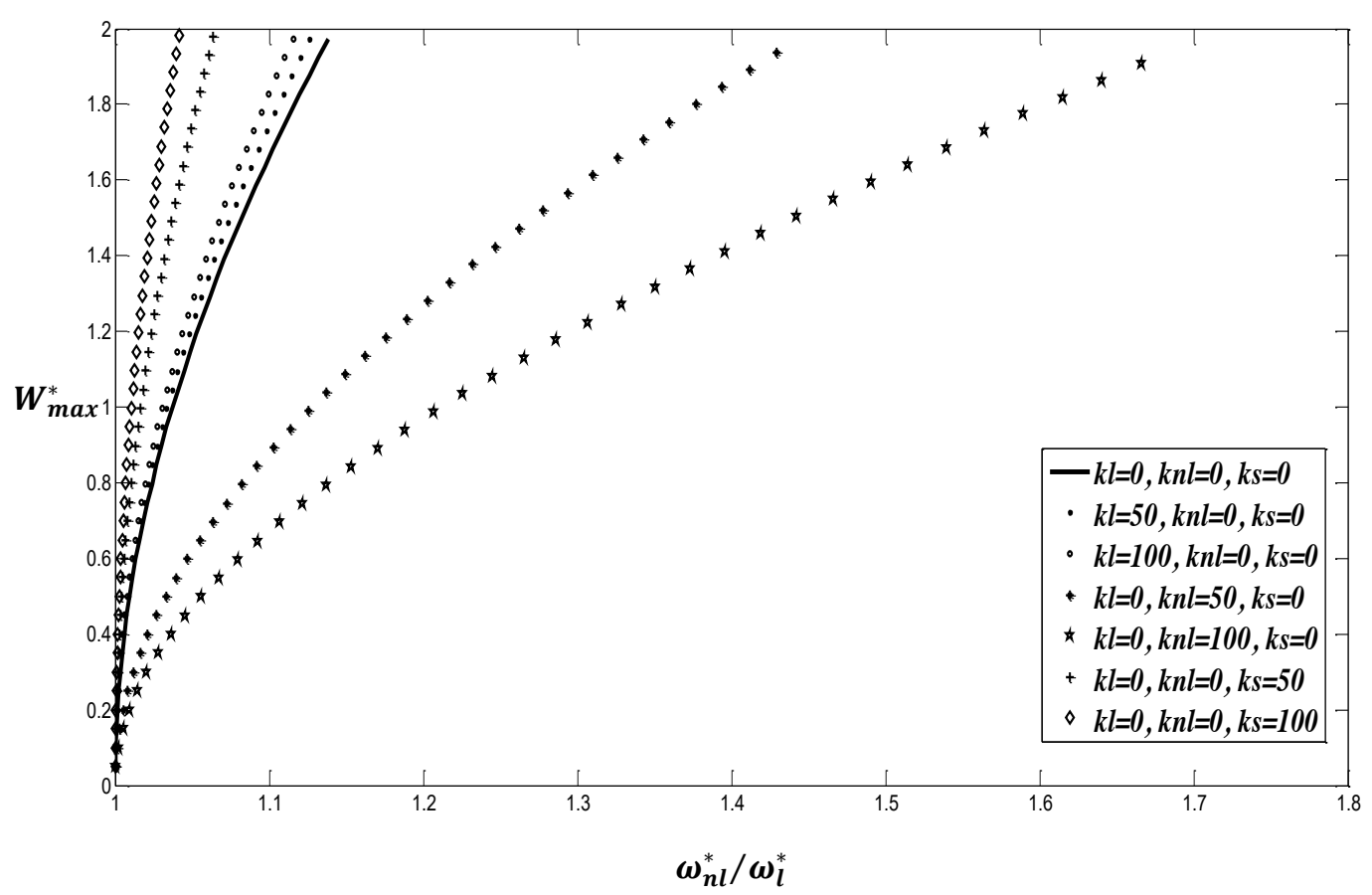

Figure 2: Effect of the elastic foundation stiffness on the frequency ratio of C-C FGM beam, case of $\mathrm{k}=2$, $\mathrm{a} / \mathrm{h}=0.3$ and $\zeta=0.2$.

It can be shown from Fig. 2 that for fixed values of a crack depth and a porosity volume fraction, the increase of linear and shear layers of the foundation leads to a decrease in the frequency ratio, whereas this ratio enhances with an increase in nonlinear elastic foundation stiffness.

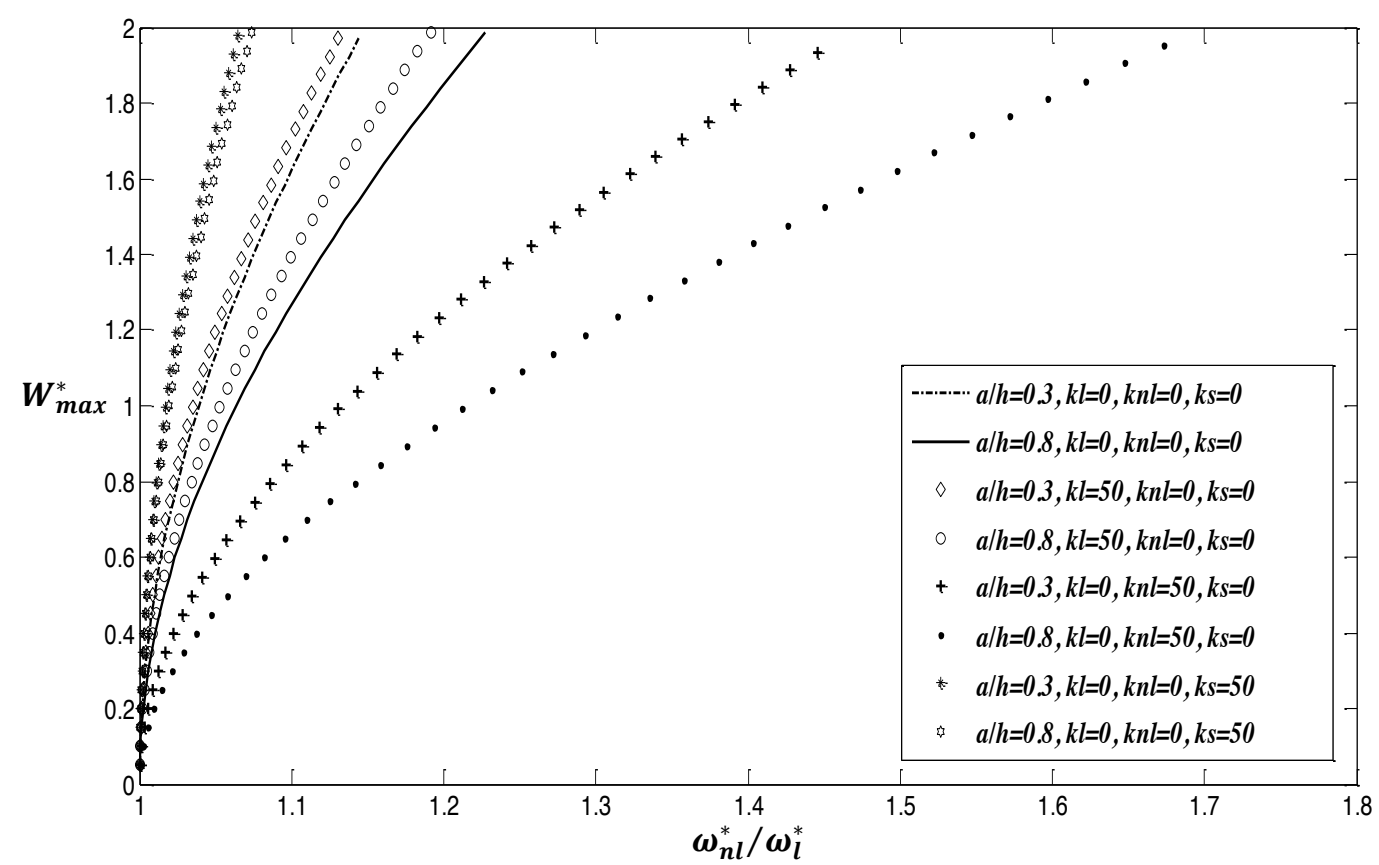

Figure 3: Effect of the elastic foundation stiffness for different crack depth, on the frequency ratio of C-C FGM beam, case of $\mathrm{k}=2$ and $\zeta=0.2$. 
It can be observed from Fig. 3 that the effect of the crack depth on the frequency ratio can be reduced by increasing the value of the linear or shear layers of the foundation respectively $k_{L}$ or $k_{S}$, this effect increases with the nonlinear elastic foundation stiffness $k_{N L}$.

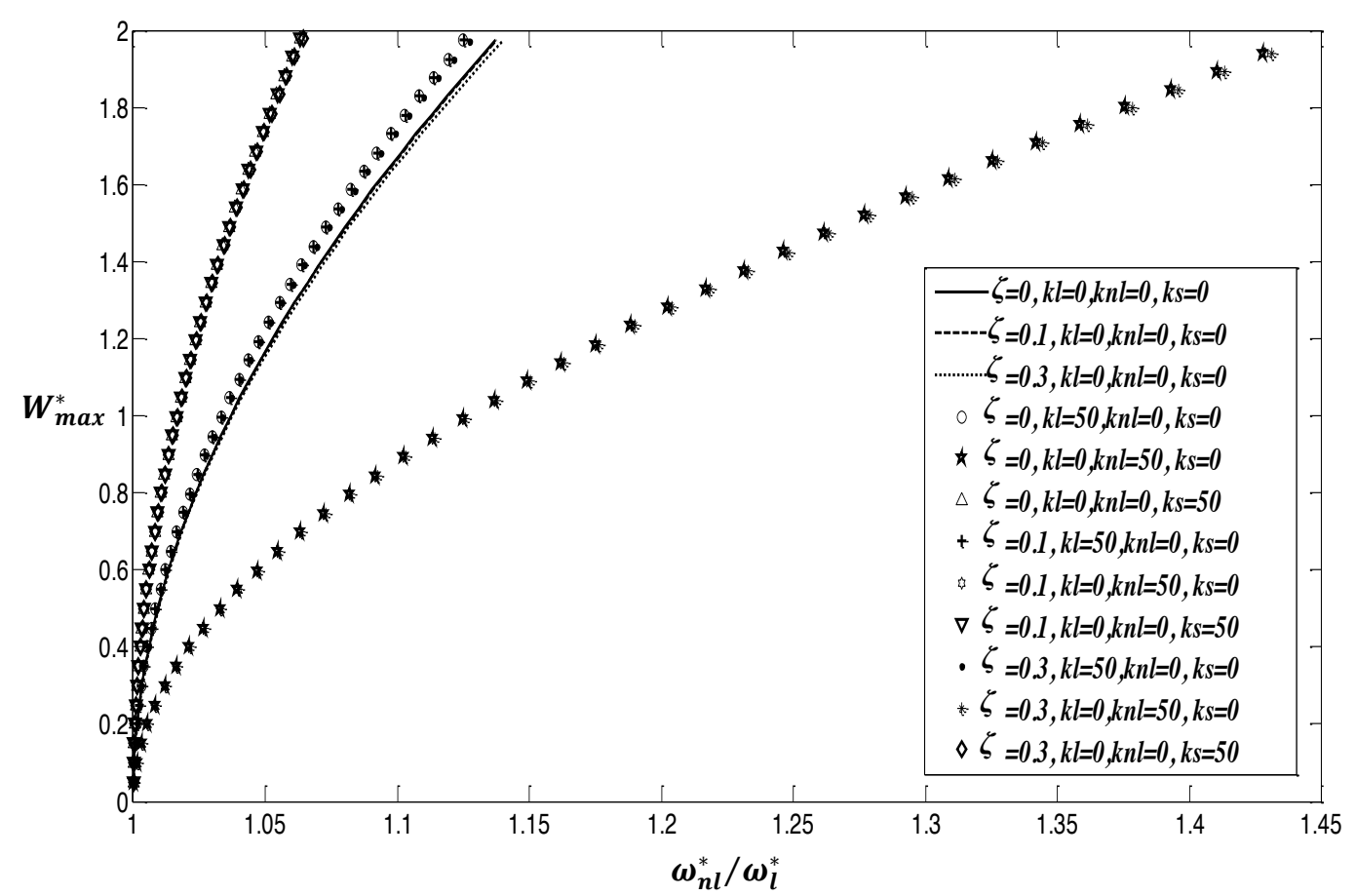

Figure 4: Effect of the elastic foundation stiffness for different porosity volume fraction, on the frequency ratio of C-C FGM beam, case of $\mathrm{k}=2$ and $\mathrm{a} / \mathrm{h}=0.3$.

It can be seen from Fig.4 that the linear and shear layer foundation parameters $k_{L}$ and $k_{S}$ are more effective in reducing the effect of porosity volume fraction than the nonlinear parameter $k_{N L}$.

\section{CONCLUSIONS}

Geometrically nonlinear free vibrations of Clamped-Clamped FGM beam with porosities containing an edge crack resting on Winkler-Pasternak foundation elastic is investigated by using Hamilton's principle and spectral analysis combined with a homogenisation method. This study is used for reduces the problem to that of isotropic homogeneous cracked beam resting on foundation elastic. Using a multimode approach, frequency ratios $\omega_{n l} / \omega_{l}$ has been calculated for several cases, the influences of the crack depth, the porosity volume fraction and foundation stiffness on the dynamic behaviour of the FGM beam are carefully examined. The accuracy of the method used in this study is investigated by comparing the results with those available from the literature. The main feature of the present contribution is the fact that can be easily treated the case of the nonlinear analysis of the cracked FGM beam with porosities resting on elastic foundation by using analytical solutions, numerical techniques and software developed for isotropic beam case.

The following conclusions are reached from the obtained results:

- The crack depth has a great influence on the frequency ratio of the FGM beam. 
- The stiffness parameter of linear and shear elastic foundation is very effective for reducing disadvantage of cracks and porosity volume fraction, this indicates that will certainly enhance the beam rigidity.

\section{REFERENCES}

[1] Ke, L., Wang, Y., Yang, J. et al. Sci. China Phys. Mech. Astron. (2012) 55: 2114. Doi: 10.1007/s11433-012-4704-y

[2] J. Yang, Y. Chen, Free vibration and buckling analyses of functionally graded beams with edge cracks, Composite Structure 83 (2008) 48-60.

[3] Yan, T., Yang, J. \& Kitipornchai, S. Nonlinear Dyn (2012) 67: 527. Doi: 10.1007/s11071011-0003-9

[4] D. Chen, J. Yang and S. Kitipornchai, Free and forced vibrations of shear deformable functionally graded porous beams, International Journal of Mechanical Sciences

[5] Al Rjoub, Y.S. \& Hamad, A.G. KSCE J Civ Eng (2016).Doi:10.1007/s12205-016-01496

[6] T. Yan, S. Kitipornchai, J. Yang, X. Q. He, Dynamic behaviour of edge cracked shear deformable functionally graded beams on an elastic foundation under a moving load, Composite Structures 93 (2011) 2992 - 3001.

[7] M. Arefi journal of Theoretical and Applied Mechanics. Volume 44, Issue 2, Pages 7182, DOI: 10.2478 / jtam-2014-0011, June 2014

[8] Akbaş, Ş.D. "FREE VIBRATION ANALYSIS OF EDGE CRACKED FUNCTIONALLY GRADED BEAMS RESTING ON WINKLER-PASTERNAK FOUNDATION." (2015): 1-15.

[9] Akbaş Ş. D. Free vibration and bending of functionally graded beams resting on elastic foundation. Res. Eng. Struct. Mat., 2015; 1: 25-37.

[10] FALLAH, A. AGHDAM, M. M. Nonlinear free vibration and post-buckling analysis of functionally graded beams on nonlinear elastic foundation. European Journal of Mechanics-A/Solids, 2011, vol. 30, no 4, p. 571-583.

[11] H. Yaghoobi, M. Torabi, Post-buckling and nonlinear free vibration analysis of geometrically imperfect functionally graded beams resting on nonlinear elastic foundation, Appl. Math. Modelling (2013), doi: http://dx.doi.org/10.1016/j.apm.2013.03.037

[12] EL Bikri K, Benamar R, Bennouna MM. Geometrically non-linear free vibrations of clamped-clamped beams with an edge crack. Compt Struct 84 (2006) 485-502. doi:10.1016/j.compstuc. 2005.09.030 